\title{
Factors predicting clinically significant fatigue in women following treatment for primary breast cancer
}

\author{
Lynn H. Gerber • Nicole Stout • Charles McGarvey • \\ Peter Soballe • Ching-yi Shieh • Guoqing Diao • \\ Barbara A. Springer • Lucinda A. Pfalzer
}

Received: 29 March 2010 / Accepted: 16 August 2010/Published online: 12 September 2010

(C) The Author(s) 2010. This article is published with open access at Springerlink.com

\begin{abstract}
Cancer-related fatigue is common, complex, and distressing. It affects $70-100 \%$ of patients receiving chemotherapy and a significant number who have completed their treatments. We assessed a number of variables in women newly diagnosed with primary breast cancer $(\mathrm{BrCa})$ to determine whether biological and/or functional measures are likely to be associated with the development of clinically significant fatigue (CSF). Two hundred twenty-three women participated in a study designed to document the impact of the diagnosis and treatment of primary breast cancer on
\end{abstract}

\author{
L. H. Gerber · G. Diao \\ George Mason University, \\ Fairfax, VA, USA \\ N. Stout $\cdot$ P. Soballe \\ National Naval Medical Center, \\ Bethesda, MD, USA \\ C. McGarvey \\ CLM Consulting, \\ Rockville, MD, USA \\ L. A. Pfalzer \\ University of Michigan, Flint, \\ Flint, MI, USA \\ L. H. Gerber · C.-y. Shieh \\ National Institutes of Health, \\ Bethesda, MD, USA \\ B. A. Springer \\ Office of the Surgeon General, \\ Rockville, MD, USA \\ L. H. Gerber $(\bowtie)$ \\ Center for the Study of Chronic Illness and Disability, \\ George Mason University, \\ Fairfax, VA, USA \\ e-mail: ngerber1@gmu.edu
}

function. Forty-four had complete data on all variables of interest at the time of confirmed diagnosis but prior to treatment (baseline) and $\geq 9$ months post-diagnosis. Objective measures and descriptive variables included history, physical examination, limb volume, hemoglobin, white blood cell count, and glucose. Patient-reported outcomes included a verbal numerical rating of fatigue $(0-10$, a score of $\geq 4$ was CSF), five subscales of the SF-36, Physical Activity Survey, and Sleep Questionnaire. At baseline, the entire cohort $(n=223)$ and the subset $(n=44)$ were not significantly different for demographic, biological, and selfreported data, except for younger age $(p=0.03)$ and ER+ $(p=0.01)$. Forty-five percent had body mass index $(\mathrm{BMI}) \geq$ $25,52 \%$ were post-menopause, and $52 \%$ received modified radical mastectomy, 39\% lumpectomy, $52 \%$ chemotherapy, $68 \%$ radiation, and $86 \%$ hormonal therapy. Number of patients with CSF increased from 1 at baseline to 11 at $\geq 9$ months of follow-up. CSF at $\geq 9$ months significantly correlated with $\mathrm{BMI} \geq 25$, abnormal white blood cell count, and increase in limb volume and inversely correlated with vigorous activity and physical function $(p<0.05)$. Fatigue increases significantly following the treatment of $\mathrm{BrCa}$. Predictors of CSF include high BMI and WBC count, increase in limb volume, and low level of physical activity. These are remediable.

Keywords Breast cancer Fatigue - Rehabilitation · Function

\section{Introduction}

Cancer-related fatigue (CRF) is a clinical entity characterized by tiredness or exhaustion not necessarily precipitated by activity; or when associated with activity, the severity 
and duration of the fatigue are out of proportion to the level of exertion [1,2]. It is distressing and persistent and interferes with mental and physical function. Fatigue is frequently experienced by patients and survivors, regardless of the type of cancer or its treatment [3, 4]. Fatigue is considered pathological when it persists for several months or is not relieved by rest. There is a wide range of its prevalence, but overall, 48\% (AHRQ Evidence Reports 02E032) of patients with cancer report fatigue. CRF is a nearly universal symptom among those receiving antineoplastic therapy [3-5]. Prevalence is particularly high in women with breast cancer ( $\mathrm{BrCa})$ [6]. CRF may result from the tumor itself or antedate its treatment. It is more likely to be a side effect of primary cancer treatment, with reports of prevalence as high as $70-100 \%$ as reported by the National Comprehensive Cancer Network. (NCCN) (http://www. nccn.org/professionals/physician_gls/f_guidelines.asp)

CRF is one of the most distressing symptoms associated with cancer and its treatment [2]. Despite the functional impact of the symptom and its relatively high frequency, its pathophysiology is still not completely understood. The predictive value of physical, functional, and laboratorymeasure abnormalities has not been demonstrated, in part because most published work is retrospective and correlative.

There have been several reports documenting associations between CRF and low hemoglobin [7], high neutrophil count [8], high circulating levels of T lymphocytes [9], elevated levels of inflammatory cytokines [10], and abnormal diurnal cortisol levels [11, 12]. This suggests that CRF has, in part, a biological basis, which may include regulation of immune function. Other studies have identified associations with sleep disturbances [13-15]. Biological factors are important contributors to CRF, but fatigue is multi-factorial and likely to be explained in terms of a bio-psychosocial and lifestyle model.

This view influenced our decision to determine whether some of the variables associated with fatigue might be predictive. In other words, a prospective approach, using repeated measures that tested some of these same variables and added measures of physical function, might establish a profile of an individual likely to develop clinically significant CRF.

Fatigue negatively influences participation in activity and exercise and has undesirable health consequences, correlated with tumor recurrence and mortality in $\mathrm{BrCa}$ patients [16-18]. In addition, aerobic exercise has been shown to be an effective treatment for mitigating CRF, as long as it targets $75 \%$ or more of heart rate for $3 \mathrm{~h} /$ week [19-24]. This is not to imply that only aerobic exercise mitigates CRF; anaerobic exercise and relaxation training have shown benefit as well [19, 20, 23-25]. This level of effort is considered exercise, rather than activity. The NCCN Practice Guidelines detail recommendations, which include exercise, for treating CRF based on published data and clinical experience (www.nccn.org).
We hypothesized that cancer survivors with CRF have both biological abnormalities, such as high body mass index (BMI) and blood counts and behavioral abnormalities such as altered physical activity and sleep. We report the findings of a prospective, repeated-measures, natural history study of women with primary $\mathrm{BrCa}$, receiving standard treatment. The study was designed to compare patient descriptors and biological measures with self-reports of fatigue, level of physical activity, and daily routines over time. Our goal was to develop a profile of individuals newly diagnosed with $\mathrm{BrCa}$ who have clinically significant cancer-related fatigue and use this to help identify who might be at considerable risk for developing clinically significant CRF.

\section{Patients and methods}

A prospective, observational IRB-approved study (NIH 02CC-0044; National Naval Medical Center (NNMC) B01-052), conducted at the NNMC Breast Care Center (Bethesda, MD) from 2001 to 2006, utilized a surveillance model to identify BrCa-related morbidity. All women newly diagnosed with unilateral, early stage $\mathrm{BrCa}$ (stages I-III) were screened by a physical therapist pre-operatively to determine eligibility and signed a consent-to-participate form. Patients were excluded if they were less than 18 years of age, had a previous history of $\mathrm{BrCa}$, bilateral $\mathrm{BrCa}$, or prior severe trauma or surgery of the upper limb (UL) on which the $\mathrm{BrCa}$ diagnosis had been made. All subjects who met the inclusion criteria and agreed to participate were consented prior to participation $(n=223)$ and were assessed at the pre-operative visit and reassessed at 1, 3, $6,9,12,18$, and 24 months post-operatively. The surveillance model clinical pathway we use is illustrated in Fig. 1. Further information was retrieved from the patients' medical records including demographic and anthropometric measures, tumor characteristics, data from routine blood draws and self-reports

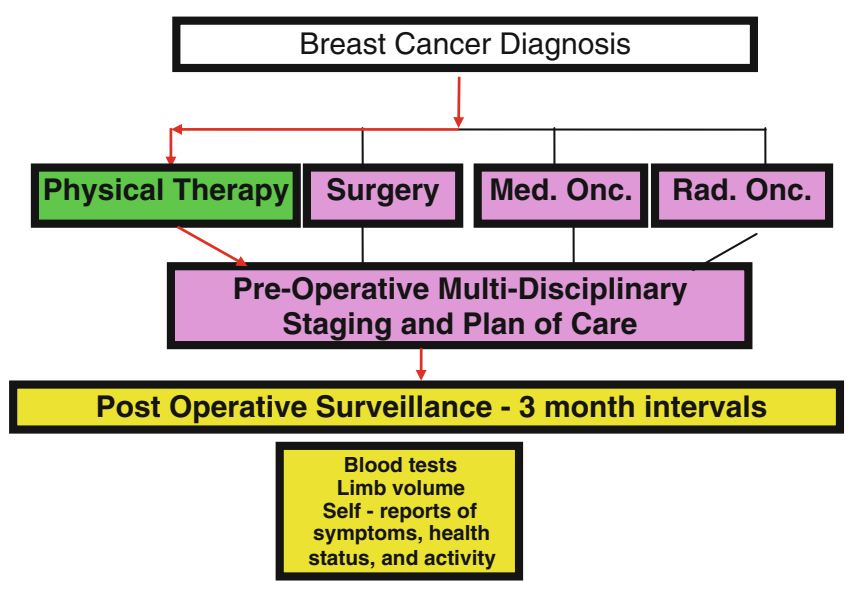

Fig. 1 Surveillance plan 
of function and physical activity. Types of treatments received are as follows: 23 received modified radical mastectomy, 17 lumpectomy, 23 chemotherapy, 30 radiation, and 20 hormonal therapy. There were 31 who had axillary nodal dissection, 12 who had sentinel node sampling, and one who had neither. Descriptive statistics for baseline findings, which were complete for 44 participants, are presented in Table 1 .

On initial visit for study purposes and at $\geq 9$-month visits, patients were asked if they experienced fatigue, and if so, they were asked to quantify verbally their level of fatigue at the time of clinic visit, scoring the intensity from 0 (no fatigue) to 10 (maximum fatigue). The fatigue measure used was a verbal numerical rating (VNR). The designation of clinically significant fatigue (CSF) was set at $\geq 4$, based on the work done by Temel, et al. [26].

At the 9-month and subsequent visits, all patients were given the Medical Outcomes Study Short Form 36 (SF-36) [27], from which we analyzed four subscales and two composite scales, including the physical function, vitality, mental function, and social function subscales and the physical function and mental function total composite. These scales have been validated, and norms have been established. A score of 50 is considered normal. Higher scores represent responses above (better than) the mean. All participants completed the Harvard Alumni Health Study Physical Activity Survey (PAS) [28, 29]. The PAS is a selfreport of the amount of time spent (in hours) performing physical activities, rated as vigorous, moderate, and mild. It was developed from epidemiological data gathered over a 40-year period from Harvard Alumni. The PAS asks respondents to enter the variety of activity in which they participate and uses an established coding system to assess the rate at which energy is expended, thereby determining the level of exercise intensity. They completed the Pittsburgh Sleep Quality Index which was used to record the number of hours spent sleeping [30].

At each visit, participants underwent optical scanning of the upper extremities to assess limb volume and possible change over time [31]. Measurements for both upper limbs were taken in a standard position with the Perometer ${ }^{\circledR} .{ }^{1} \mathrm{UL}$ volume was calculated using $80 \%$ of the total limb length, measured from the ulnar styloid process to the tip of the acromion, for standardization. Body weight was taken at each visit to control for weight change. Diagnostic criteria for lymphedema was a volume increase in the affected UL volume of at least $3 \%\left(100 \mathrm{~cm}^{3}\right)$, compared to the subject's pre-operative measurement and with consideration of the contralateral limb volume changes. The technique used was that reported in a previous study [31, 32].

\footnotetext{
${ }^{1}$ Perosystem, Messegerate, Wuppertal, Germany
}

\section{Data collection}

All subjects were recruited from NNMC-BCC. Subjects were interviewed and evaluated by a physical therapist for their initial (baseline) examination and for all follow-up appointments. There were 223 subjects recruited to the study; however, over the 5-year period of follow-up, only 44 had adequate follow-up data to be included. Table 1 displays the variables of interest for the entire group $(n=233)$ and the subset we used for the analysis $(n=44)$.

Statistical analyses

Analyses were performed on all 44 subjects for whom we had complete data sets of the variables of interest. Spearman's correlation coefficients were used in all correlation analyses, given the relatively small sample size and that some of the variables are highly skewed. Correlation analyses were performed between CSF, defined as $\geq 4$ on the VNR, and variables of interest. We performed logistic regression analyses to assess the effects of baseline demographic and biological variables on the VNR at 9 months and after. We also performed simple linear regression analyses to assess the effects of baseline demographic variables and biological variables at 9 months and after and on variables which measured function, including vigorous activity (PAS), sleeping (PSQI), physical function, mental health, vitality, and social function (SF-36). For all regression analyses, we used forward model selection to select a model such that no other variable met the 0.1 significance level for entry into the model. All data analyses were conducted using SAS v 9.2.

\section{Results}

Forty-four women, out of a total population of 223 who had enrolled in the study, had complete baseline and follow-up data collected between 9 and 24 months available for analysis of descriptive variables of interest (Table 1). All baseline data available for the entire cohort and that which were complete and acceptable for analysis for the follow-up period are presented in Table 1 . There were statistically significant $(p<0.05)$ differences in the variables for which comparisons could be made between the entire cohort $(n=233)$ and the subjects reported in this study $(n=44)$. Statistically significant differences in the two groups were demonstrated for age (study cohort is younger) and ER positivity (study cohort has a greater number in this group). Follow-up data were required to have been collected after completion of primary treatment of $\mathrm{BrCa}$.

Table 2 presents data on variables of interest and their relationship to the presence of clinically significant fatigue 


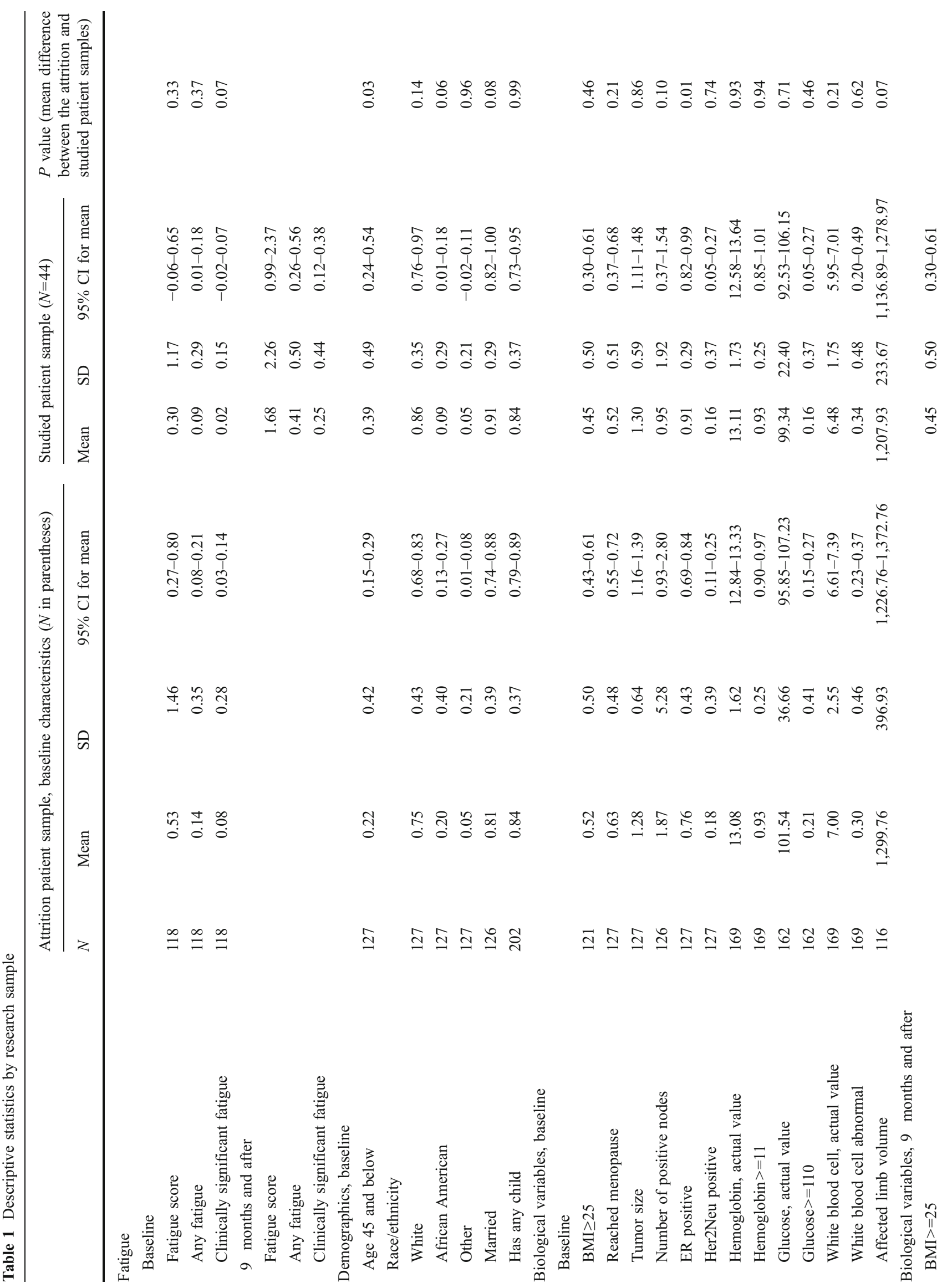




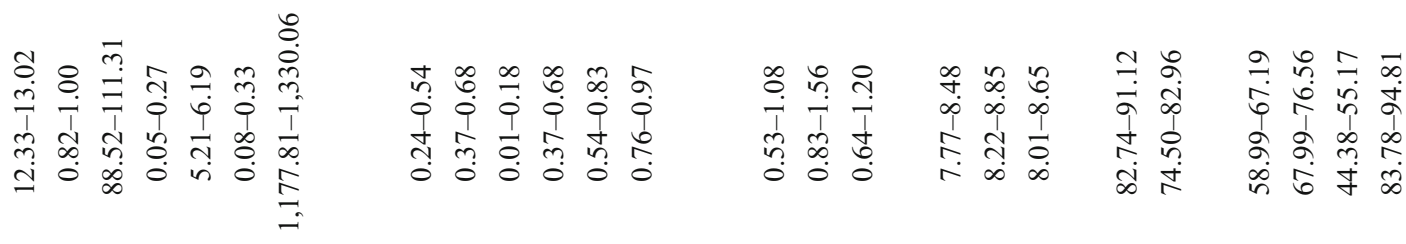

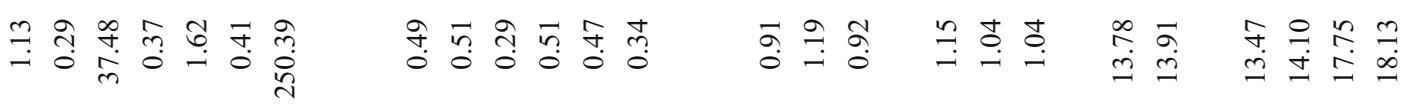

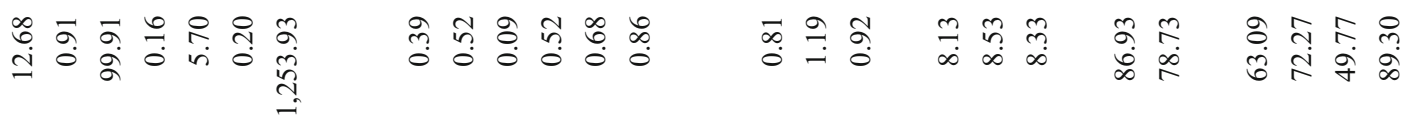

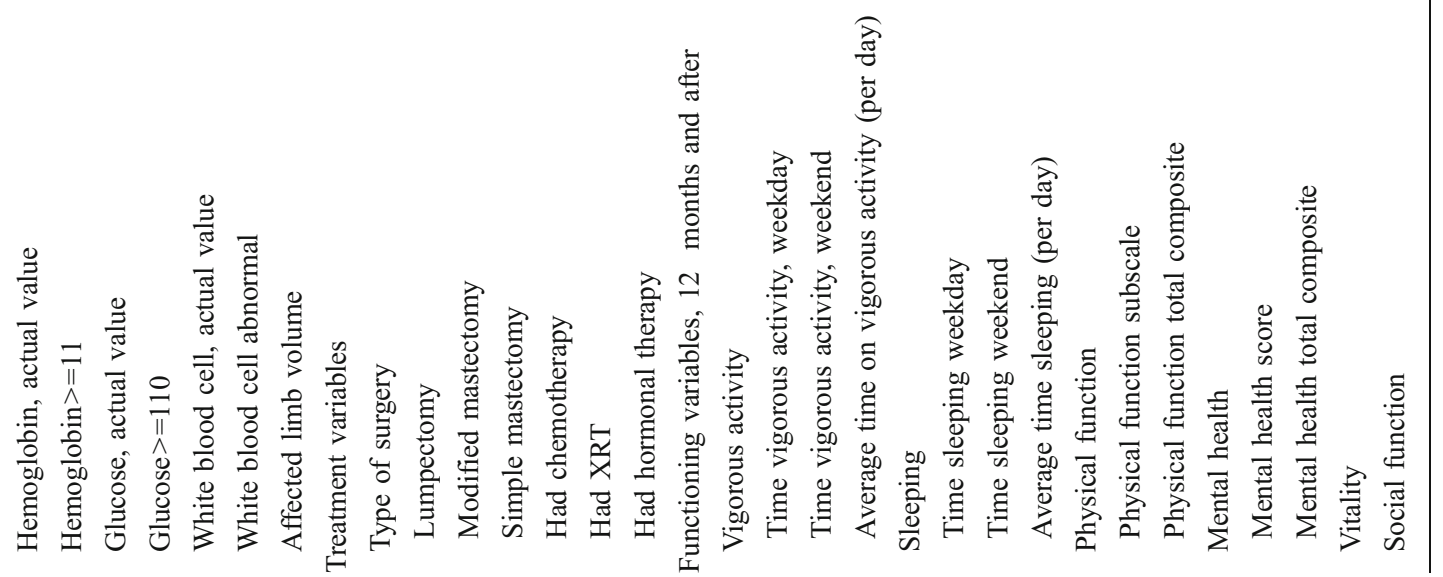


Table 2 Correlation between selected variables and clinically significant fatigue at $\geq 9$ months after baseline measures

\begin{tabular}{|c|c|}
\hline$N=44$ & Coefficient \\
\hline \multicolumn{2}{|l|}{ Demographics, baseline } \\
\hline Age 45 and below & -0.243 \\
\hline \multicolumn{2}{|l|}{ Race/ethnicity } \\
\hline White & 0.076 \\
\hline African American & 0.000 \\
\hline Other & -0.126 \\
\hline Married & 0.000 \\
\hline Has any child & 0.108 \\
\hline \multicolumn{2}{|l|}{ Biological variables, baseline } \\
\hline \multicolumn{2}{|l|}{ Baseline } \\
\hline $\mathrm{BMI}>=25$ & $0.422 * * *$ \\
\hline Reached menopause & 0.236 \\
\hline Tumor size & 0.050 \\
\hline Number of positive nodes & $-0.308 * *$ \\
\hline ER positive & -0.183 \\
\hline Her2Neu positive & -0.108 \\
\hline Hemoglobin, actual value & 0.039 \\
\hline Hemoglobin $>=11$ & 0.156 \\
\hline Glucose, actual value & 0.197 \\
\hline Glucose $>=110$ & 0.036 \\
\hline White blood cell, actual value & $0.432 * * *$ \\
\hline White blood cell abnormal & $0.360 * *$ \\
\hline Affected limb volume & -0.027 \\
\hline \multicolumn{2}{|l|}{ Biological variables, 9 months and after } \\
\hline $\mathrm{BMI}>=25$ & $0.316^{* *}$ \\
\hline Hemoglobin, actual value & -0.079 \\
\hline Hemoglobin $>=11$ & -0.183 \\
\hline Glucose, actual value & 0.172 \\
\hline Glucose $>=110$ & 0.036 \\
\hline White blood cell, actual value & $0.382 * *$ \\
\hline White blood cell abnormal & -0.033 \\
\hline Affected limb volume & 0.188 \\
\hline Limb volume increase $100 \mathrm{cc}$ or more & $0.329 * *$ \\
\hline \multicolumn{2}{|l|}{ Treatment variables } \\
\hline \multicolumn{2}{|l|}{ Type of surgery } \\
\hline Lumpectomy & 0.189 \\
\hline Modified mastectomy & -0.183 \\
\hline Simple mastectomy & 0.000 \\
\hline Had chemotherapy & -0.079 \\
\hline Had XRT & -0.056 \\
\hline Had hormonal therapy & -0.229 \\
\hline \multicolumn{2}{|l|}{ Functioning variables, 12 months and after } \\
\hline \multicolumn{2}{|l|}{ Vigorous activity } \\
\hline Time vigorous activity, weekday & $-0.314 * *$ \\
\hline Time vigorous activity, weekend & -0.125 \\
\hline Average time on vigorous activity (per day) & $-0.259 *$ \\
\hline \multicolumn{2}{|l|}{ Sleeping } \\
\hline Time sleeping weekday & 0.063 \\
\hline
\end{tabular}

Table 2 (continued)

\begin{tabular}{lc}
\hline$N=44$ & Coefficient \\
\hline Time sleeping weekend & -0.036 \\
Average time sleeping (per day) & 0.051 \\
Physical function & $-0.302^{* *}$ \\
Physical function subscale & -0.161 \\
Physical function total composite & \\
Mental health & 0.125 \\
Mental health score & -0.147 \\
Mental health total composite & -0.176 \\
Vitality & 0.106 \\
Social function
\end{tabular}

Spearman correlation coefficients presented in the table. Clinically significant fatigue is defined as fatigue score $\geq 4$

${ }^{*} p \geq 0.10 ; * * p<0.05 ; * * * p<0.01 ; * * * * p<0.001$

at $\geq 9$ months following diagnosis. Breast cancer treatments are included, but none was significantly correlated with CSF. We performed a logistic regression analysis on these data. Entry into the model required statistical significance for the variable at $p \leq 0.10$. The following remained significant: menopausal status (odds ratio (OR) 10.65, $p=$ 0.03 ), limb volume increase (OR for $100-\mathrm{cm}^{3}$ increase of limb volume is $3.75, p=0.02$ ), increased white blood cell (WBC; OR 1.99, $p=0.04$ ). The amount of time spent sleeping did not differ significantly between those with CSF $(n=11)$ and those without $(n=33)$. The average for the CSF group was $8.27 \mathrm{~h}$ per night and, for those without, $8.35 \mathrm{~h}$ per night.

Table 3 compares the variables of interest with selected subscales of the SF-36. The analysis was performed, in part, to contrast the relationships among self-reported vitality, physical and mental status, and the variables of interest. Vitality is often considered the inverse of fatigue and often reported with fatigue measures. We performed a linear regression analysis on these data. Entry into the model required statistical significance for the variable at $p=<0.10$. The following remained significant and were all inversely related: having children $(p=0.06)$, menopausal status $(p=0.01)$, and BMI $(p=0.01)$.

\section{Discussion}

The prospective, descriptive study from which these data were collected was designed to identify the prevalence of and risk factors for upper limb impairments and functional limitations in women with the diagnosis of primary $\mathrm{BrCa}$. The study employed a surveillance model with repeated measures over a 5-year period to enable identification of variables the investigators believed would influence func- 


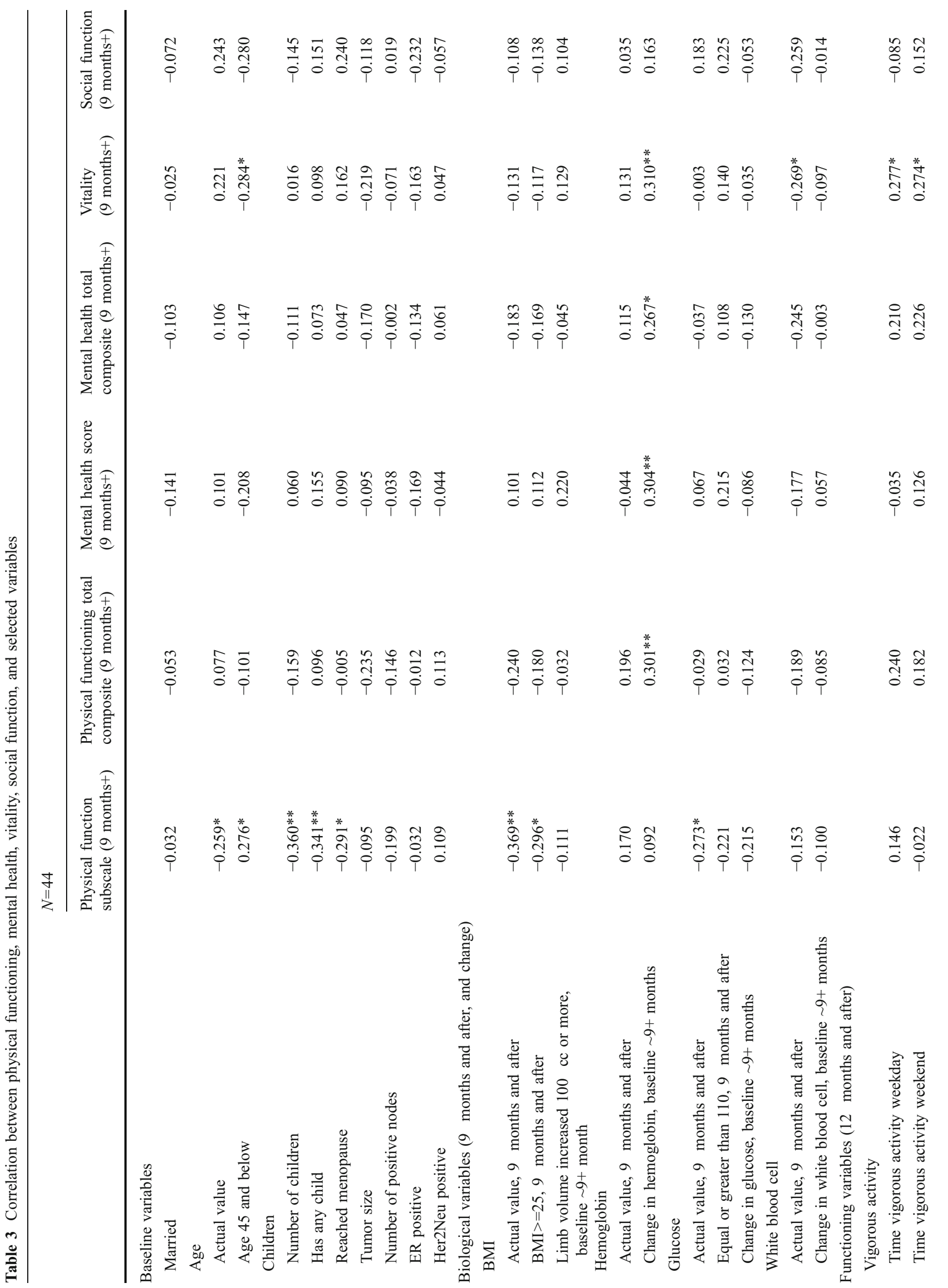




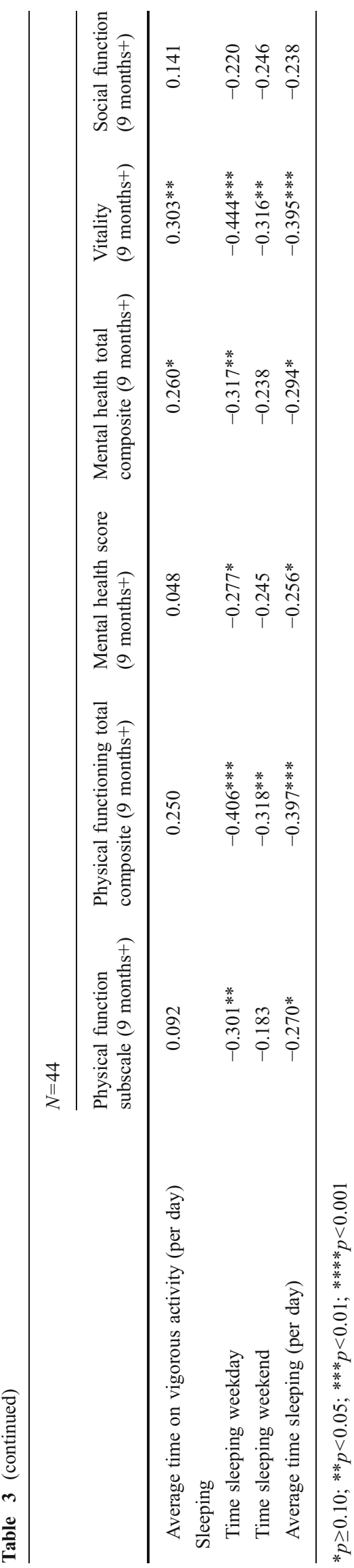

tion over time. This approach was used to record at specified intervals the signs and symptoms of physical impairment, functional limitation, and fatigue. Previously reported work using this model demonstrates that prospective surveillance and early intervention in women with breast cancer resulted in successful identification and treatment of lymphedema [32].

The measurement of fatigue has posed significant methodological difficulties for investigators. There is no consensus on which measure should be used, although recent opinions have been published that propose criteria for what constitutes acceptable fatigue measures; and several have been shown to be particularly suitable for evaluating cancer patients [33-35]. Frequently, health care providers do not assess patients for the presence of fatigue or seek to determine contributors to fatigue and often do not prescribe effective treatments [36]. Effective treatment begins with the identification of the undesirable process or syndrome and includes determination of its possible contributors.

We chose to use a unidimensional verbal report of fatigue because it was easy to administer in a busy clinic. It is sensitive to change and, although not specific to CRF, has been validated against commonly used fatigue measures in cancer patients and patients with rheumatic disease [26, 37]. The selection of a value of " 4 " as clinically significant was based on the NCCN guidelines and has been confirmed as being valid [26]. We also used a measure of vitality, included as a subscale of the SF-36, which has been used frequently as an indicator of fatigue in cancer survivors. This is a self-report about whether an individual feels "energetic." Some investigators have suggested that this scale measures central fatigue and reflects a combination of mood and motivation and is not a strong indicator of either peripheral fatigue or aerobic fitness [38]. In the study reported here, we demonstrate correlation between high vitality scores and less sleep, and increase in hemoglobin and vigorous activity.

Patients in this cohort report a $25 \%$ incidence of CSF at least 9 months after the diagnosis of $\mathrm{BrCa}$ is made and confirm the observation that fatigue in cancer survivors is prevalent and persists after primary treatment is completed. The findings are somewhat lower than several studies reporting CRF but are generally consistent with the published experience of other investigators [1-7, 39]. Explanations for these differences include the relatively low average age of this cohort (50.5 years) and some are active duty military or military dependents and many are in the work force.

This study reports data that correlates of persistent clinically significant $\mathrm{CRF}$ include number of positive nodes, increase in limb volume, high BMI, an increase in WBC count over baseline to $>8,000$, and low level of physical activity and physical function. We extend these observations and suggest that a $\mathrm{BMI} \geq 25, \mathrm{WBC}>8,000$, 
and increase in limb volume are predictive of fatigue. This study does not establish the causal relationship among these variables and CSF in cancer survivors, rather it establishes that women likely to have CSF following BrCa diagnosis and treatment are likely to demonstrate these findings.

CRF has been shown to correlate with several factors, including anemia, inflammatory cytokines, cortisol, and obesity [7-17, 40-43]. We included some of these variables for measurement in this study and added outcomes that measure physical activity and function. The rationale behind this is that physical activity, particularly strenuous physical activity ( $>$ 5METS), is likely to have a beneficial outcome for breast cancer patients. Women who do not participate in strenuous physical activity have been shown to have less good clinical outcomes. Exercise has been associated with improved outcomes in women with $\mathrm{BrCa}[17,19-21,44,45]$ and has been demonstrated to have a significant effect in improving CRF [21-25] and also has been shown to improve glucose uptake and insulin sensitivity [16]. Possible mechanisms for decreased prevalence of CRF and good health outcomes in women with $\mathrm{BrCa}$ who are physically active pertain to the effect of exercise and activity on a variety of critical pathways that impact metabolism and inflammation. These include decrease in serum pro-inflammatory cytokines [41], mobilization of visceral fat, and increased insulin sensitivity and glucose uptake [16]. These are of specific benefit to women with $\mathrm{BrCa}$ diagnoses because of the adverse associations between obesity and $\mathrm{BrCa}[17,18]$, and recurrence and mortality from $\mathrm{BrCa}$ associated with obesity [18]. Physical activity has been reported to help regulate sleep and diurnal variation of cortisol [46-49]. The amount of time spent sleeping was not significantly different between the two groups in this study.

The data summarized in Table 3 were selected to demonstrate which functional measures (physical, mental, or vitality) correlate with the variables of interest. Additionally, we included these data to present comparative data among the subscales of the SF-36 but also to point out differences between the vitality subscale and the others. Of note, the less time spent sleeping, the higher are the subscale scores on the SF-36.

The focus of this paper is to identify clinical and biological variables likely to be associated with persistent, clinically significant fatigue in $\mathrm{BrCa}$ survivors. However, it is tempting to cluster the findings reported here into a speculative hypothesis that those with CSF may have a proinflammatory state. The high BMI and its increased burden of inflammatory adipocytes and the elevated WBC, combined with an increase in limb edema, may all be contributing to an inflammatory state. This concept has been supported by publications of retrospective studies in which pro-inflammatory cytokines are associated with the presence of CRF $[10,40,50]$. Lymphedema may be an inflammatory transudate, and the fluid itself may be high in pro-inflammatory cytokines [51]. These inflammatory cytokines have been implicated in other illnesses associated with fatigue, some of which have affective components, as well $[52,53]$. The "clustering" of BMI, limb edema, and elevation of WBC reported here suggests that inflammatory as well as metabolic factors may be contributing to CRF.

Other investigators have established an important role for the presence of low hemoglobin in CRF [7, 54]. We did not find a correlation between a decrease in hemoglobin and fatigue. We did identify a significant benefit to patients who had a rise in hemoglobin from baseline, as measured by physical, mental, and vitality scores of the SF-36 subscales.

Many of the variables we chose to explore in this study have been shown to be contributors to $\mathrm{CRF}$ in the $\mathrm{BrCa}$ population [55]. We confirm some and add to these predictors including number of positive lymph nodes, reaching menopause, presence of high BMI, increase in limb volume, increase in $\mathrm{WBC}$, and low level of vigorous and physical activity. The regression analysis of these data suggest that the presence of menopause, increase in WBC count, and limb volume of $>100 \mathrm{~cm}^{3}$ are significant predictors for CSF fatigue in this population. Several of the abnormalities identified here, which are associated with low levels of activity, can be addressed through rehabilitation and medical management to prevent, mitigate, or reverse CSF.

There are several limitations to the study we report here. We recruited 223 participants in this prospective study. We report complete data on 44 . The fact that we have repeated measures on all variables in only $22 \%$ is a result of a number of factors: (a) some did not complete the original baseline data; (b) this group of subjects is a highly mobile population due to military deployment and transfer to other locations; (c) completion of the questionnaires was time consuming and patients were often busy seeing multiple providers at the same clinic setting.

We have tried to address the issue of dropouts by presenting and analyzing the data in such a way that comparisons can be made between the entire cohort and the subgroup of 44 in the final cohort. The whole group is similar to the subset we report on all variables reported, as we displayed in Table 1.

Another difficulty with the study is that there is a small number of participants, but we are interested in a large number of variables, across many domains, including anthropometric and biological measures, demographic descriptors, activity level, and sleep. In order to reduce the likelihood of bias, we used a prospective design and non-parametric, Spearman rank order statistical analytic approach. We were fortunate to have been able to evaluate and treat patients in the same setting, with only a few care providers utilizing a standard staging procedure and offer patients a prescribed regimen based upon their $\mathrm{BrCa}$ stage. 
Another difficulty we faced was the ability to capture baseline data at a time when subjects were experiencing a difficult time and facing uncertainty about their treatments and future. Further, this occurred in a busy clinic. We selected a unidimensional measure of fatigue, which has its limitations. However, it has validity in cancer patients [26], and its utility in identifying people with clinically significant fatigue has been accepted. Finally, unidimensional fatigue measures have been used to determine who needs treatment in populations with cancer diagnoses.

\section{Conclusion}

A surveillance approach, using valid instruments and repeated measures in patients with primary $\mathrm{BrCa}$, is one method that can be employed to identify clinically significant CRF symptoms throughout the course of evaluation and treatment of $\mathrm{BrCa}$. The patient most likely to benefit from this approach because of an increased risk for developing CRF and CSF is one who is post-menopause and overweight and has an increase in limb volume of more than $100 \mathrm{~cm}^{3}$ from baseline measures and an elevation in WBC count.

Acknowledgements This research was supported with intramural research funds of the Clinical Research Center, the National Institutes of Health, Bethesda, Maryland. In addition, support for students engaged in this research from Mrs. Marcia Di Trapani and the Dominion Guild. We would like to acknowledge valuable assistance from Ms. Angela Corriveau and Lynn Fan, graduate students in the College of Health and Human Services, George Mason University.

Conflict of interest I certify that I have no conflict of interest relevant to this manuscript.

Open Access This article is distributed under the terms of the Creative Commons Attribution Noncommercial License which permits any noncommercial use, distribution, and reproduction in any medium, provided the original author(s) and source are credited.

\section{References}

1. Cella D, Davis K, Breitbart W et al (2001) Cancer related fatigue: prevalence of proposed diagnostic criteria in a United States sampling of cancer survivors. J Clin Oncol 19:3385-3391

2. Hofman M, Ryan JL, Figueora-Moseley CD et al (2007) Cancer related fatigue: scale of the problem. Oncologist 12(Suppl 1):4-10

3. Bower JE (2005) Prevalence and causes of fatigue in long-term breast carcinoma survivors: a longitudinal investigation. Cancer 106:751-758

4. Curt GA (2000) Impact of fatigue on quality of life in oncology patients. Semin Hematol 37(4 suppl 6):14-17

5. Nieboer P, Buijsc RS et al (2005) Fatigue and relating factors in high risk breast cancer patients treated with adjuvant standard-or high dose chemo therapy. J Clin Oncol 23:8296-8304

6. Wagner LI, Cella D (2004) Fatigue and cancer: causes, prevalence and treatment approaches. Br J Cancer 91:822-828
7. Ludwig H, Strasser K (2001) Symptomatology of anemia. Semin Oncol 28:7-14

8. Gripp S, Moeller S, Bolke E et al (2007) Survival predictors in terminally ill cancer patients by clinical estimates, laboratory tests and self-reported anxiety and depression. J Clin Oncol 25:3313-3320

9. Bower JE, Ganz PA, Aziz N et al (2003) T cell homeostasis in breast cancer survivors with persistent fatigue. J Natl Cancer Inst 95(15): 116508

10. Collado-Hidalgo A, Bower JE, Ganz PA, Cole SW, Irwin MR (2006) Inflammatory biomarkers for persistent fatigue in breast cancer survivors. Clin Cancer Res 12(9):2759-2766

11. Bower JE, Ganz PA, Aziz N et al (2007) Inflammatory response to psychological stress in fatigued breast cancer survivors: relationship to glucocorticosteroids. J Brain Behav Immun 21(3):251-258

12. Bower JE, Ganz PA, Aziz N (2005) Altered cortisol response to psychological stress in breast cancer survivors with persistent fatigue. Psychosom Med 67(2):277-280

13. Ancoli-Israel S (2001) The relationship between fatigue \& sleep in cancer patients: a review. Eur J Cancer Care 10:245-255

14. Berger AM, Farr LA, Kuhn BR et al (2007) Values of sleep/wake, activity/rest, circadian rhythm and fatigue prior to adjuvant breast cancer chemotherapy. J Pain Symptom Manage 33(4):398-409

15. Roscoe JA, Kaufman ME, Matteson-Rusby SE et al (2007) Cancer related fatigue \& sleep disorders. The Onclogist 12(Suppl 1): $35-42$

16. Goodwin PJ, Ennis M, Pritchard KI et al (2002) Fasting insulin and outcome in early-stage breast cancer: results of a prospective cohort study. J Clin Oncol 20:42-51

17. Lorincz AM, Sukumar S (2006) Molecular links between obesity and breast cancer. Endocr Relat Cancer 13(2):279-292

18. Calle EE, Rodriguez C, Walker-Thurmond K, Thun MJ (2003) Overweight, obesity, and mortality from cancer in a prospectively studied cohort of U.S. adults. N Engl J Med 348(17):1625-1638

19. Dimeo FC (2004) Effect of aerobic exercise and relaxation on fatigue and physical performance of cancer patients after surgery. J Supportive Care Cancer 12:774-779

20. Courneya KS (2007) Effects of aerobic and resistance exercise in breast cancer patients receiving adjuvant chemotherapy: a multicenter randomized controlled trial. J Clin Oncology 25:4396-4404

21. Mock V, Frangakis C, Davidson NE et al (2004) Exercise manages fatigue during breast cancer treatment: a randomized control trial. Psycho-oncol 14:464-477

22. Hewitt JA, Mikbel K, van Someren Ka et al (2005) Exercise for breast cancer survival: the effect on cancer risk and cancer related fatigue. Int J Fertil Womens Med 50(Pt1):231-239

23. McNeely ML, Campbell KL, Rowe BH et al (2006) Effects of exercise on breast cancer patients and survivors: a systematic review and meta-analysis. CMAJ 175:34-41

24. Courneya KS (2003) Exercise in cancer survivors: an overview of research. Med Sci Sports Exerc 35(11):1846-1852

25. Schmitz KH, Ahmed RL, Hannan PJ, Yee D (2005) Safety and efficacy of weight training in recent breast cancer survivors to alter body composition, insulin, and insulin-like growth factor axis proteins. Cancer Epidemiol Biomark Prev 14(7):1672-1680

26. Temel JS, Pirl WF, Reclitis CJ et al (2006) Feasibility and validity of a one-item fatigue screen in a thoracic oncology clinic. J Thor Oncol 1:454-459

27. Stewart AL, Hays RD, Ware JE (1988) The MOS short form general health survey: reliability and validity in a patient population. Med Care 26:724-735

28. Ainsworth BE, Leon AS, Richardson MT et al (1993) Accuracy of the college alumnus physical activity questionnaire. J Clin Epidemiol 46(12):1403-1411

29. Paffenbarger RS Jr, Blair SN, Lee IM, Hyde RT (1993) Measurement of physical activity to assess health effects in freeliving populations. Med Sci Sports Exerc 25(1):60-70 
30. Buysse DJ, Reynolds CF 3rd, Monk TH (1989) The Pittsburgh sleep quality index: a new instrument for psychiatric practice and research. Psychiatry Res 28(2):193-213

31. Stanton AW, Northfield JW, Holroyd B et al (1997) Validation of an optoelectronic limb volumeter (Perometer). Lymphology 30:77-97

32. Stout Gergich NL, Pfalzer LA, McGarvey CL et al (2008) Preoperative assessment enables the early diagnosis and successful treatment of lymphedema. Cancer 112:2809-2819

33. Yellen SB, Cella DF, Webster K, Blendowski C, Kaplan E (1997) Measuring fatigue and other anemia-related symptoms with the Functional Assessment of Cancer Therapy (FACT) measurement system. J Pain Symptom Manage 13:63-74

34. Minton O, Stone P (2009) A systematic review of the scales used for the measurement of cancer-related fatigue (CRF). Ann Oncol 20:17-25

35. Jacobsen PB (2004) Assessment of fatigue in cancer patients. J Natl Cancer Inst Monogr 32:93-97

36. Gledhill J (2005) A qualitative study of the characteristics and representations of fatigue in a French speaking population of cancer patients and healthy subjects. Eur J Oncol 9:294-312

37. Pouchot J, Kherani RB, Brant R et al (2008) Determination of the minimal clinically important difference for seven fatigue measures in rheumatoid arthritis. J Clin Epidemiol 61:705-713

38. Weinstein AA, Drinkard BM, Diao G, Furst G, Dale JK, Straus SE, Gerber LH (2009) Exploratory analysis of the relationships between aerobic capacity and self-reported fatigue in patients with rheumatoid arthritis, polymyositis, and chronic fatigue syndrome. PM R 1(7):620-628

39. Winningham ML, Nail LM, Burke MB et al (1994) Fatigue and the cancer experience: the state of the knowledge. Oncol Nurs Forum 21(1):23-36

40. Demark-Wahnefried W et al (1997) Reduced rates of metabolism and decreased physical activity in breast cancer patients receiving adjuvant chemotherapy. Am J Clin Nutr 65:1495-1501

41. Bower JE, Ganz PA, Aziz N, Fahey JL (2002) Fatigue and proinflammatory cytokine activity in breast cancer survivors. Psychosom Med 64:604-611

42. Karayiannakis AJ, Syrigo KN, Polychonides A et al (2001) Serum levels of tumor necrosis factor and nutritional status in pancreatic cancer patients. Anticancer Res 21:1355-1358
43. Basen-Engquist K, Scruggs S, Jhingran A, Bodurka DC, Lu K, Ramondetta L, Hughes D, Carmack Taylor C (2009) Physical activity and obesity in endometrial cancer survivors: associations with pain, fatigue, and physical functioning. Am J Obstet Gynecol 200(3):288.e1-288.e8

44. Holick CN, Newcomb P, Trentham-Dietz A (2008) Physical activity and survival after diagnosis of invasive breast cancer. Cancer Epidemiol Biomark Prev 17(2):379-386

45. Holmes MD, Chen WY, Feskanich D, Kroenke CH, Colditz GA (2005) Physical activity and survival after breast cancer diagnosis. JAMA 293:2479-2486

46. Sherrill DL, Kotchou K, Quan SF (1998) Association of physical activity and human sleep disorders. Arch Intern Med 158:1894 1898

47. Singh NA, Clements KM, Fiatarone MA (1997) A randomized controlled trial of the effect of exercise on sleep. Sleep 20:95-101

48. King AC, Oman RF, Brassington GS, Bliwise DL, Haskell WL (1997) Moderate-intensity exercise and self-rated quality of sleep in older adults. A randomized controlled trial. JAMA 277:32-37

49. Yeh GY, Mietus JE, Peng CK et al (2008) Enhancement of sleep stability with Tai Chi exercise in chronic heart failure: preliminary findings using an ECG-based spectrogram method. Sleep Med 9:527-536

50. Jager A, Sleijfer S (2008) The pathogenesis of CRF, could increased activity of the proinflammatory cytokines be the culprit? J Euro Ca 44:174-180

51. Mak S, Yeo W, Mo KF et al (2007) A case control study on risk factors of lymphedema after axillary lymph node dissection for breast cancer in Hong Kong 25(18S):948

52. Heesen C, Nawrath L, Reich C et al (2006) Fatigue in MS: an example of cytokine mediated sickness behavior. J Neural Neuro Surg Psych 77:34-39

53. Skowera A, Cleare A, Blair D et al (2004) High levels of type 2 cytokine producing cells in chronic fatigue syndrome. Clin Exp Immunol 135:294-302

54. Cella D (1998) Factors influencing quality of life in cancer patients: anemia and fatigue. Semin Oncol 25:43-46

55. Wielgus KK, Berger AM, Hertzog M (2009) Predictors of fatigue 30 days after completing anthracycline plus taxane adjuvant chemotherapy for breast cancer. Oncol Nurs Forum 36(1):38 